\title{
POLÍTICAS PÚBLICAS: PROVISIÓN DE BIENESTAR. CRITERIOS DE SOSTENIBILIDAD
}

\section{PUBLIC POLICIES: WELL-BEING PROVISION SUSTAINABILITY CRITERIA}

\author{
Gustavo Zaragoza Pascual \\ Director General de Análisis de Políticas Públicas y Coordinación \\ Generalitat Valenciana
}

\begin{abstract}
Resumen: Este artículo reflexiona sobre la importancia que las prácticas de intervención comunitaria y mediación han tenido sobre los sistemas de protección social, y explica la aparición y expansión del Estado de bienestar, un logro relativamente reciente en nuestro país que se ha ido configurando durante los últimos 35 años, dibujando un modelo propio, coincidente en diversos aspectos, con el que se ha desarrollado en el resto de países de la Europa meridional. Esta fórmula o modelo mediterráneo, ha supuesto un avance considerable en las condiciones de vida de los ciudadanos, aunque actualmente, se encuentra sometida a numerosos dilemas e incertidumbres, especialmente relacionados con la capacidad e intensidad protectora y sus consecuencias en capas amplias de la sociedad. También se analizan los elementos necesarios para garantizar la sostenibilidad de las políticas públicas de bienestar y las recomendaciones de adaptación a los cambios sociodemográficos producidos en el último tramo del siglo XX.
\end{abstract}

Palabras Clave: Intervención Social, Intervención Comunitaria, Mediación, Estado de Bienestar, Sistemas de Protección Social, Servicios Sociales, Sostenibilidad.

Abstract: The aim of this article is a reflection about the importance of practices, community intervention and mediation in the systems of social protection. With this purpose makes a review about the apparition and expansion of the known like State of welfare, a relatively recent attainment in our country that has gone configuring during the last 35 years, drawing an own model, coincident in diverse appearances, with which has developed in the rest of countries of the meridional Europe. This formula or Mediterranean model, has supposed a considerable advance in the conditions of life of the citizens, in spite of which, at present, finds subjected to numerous dilemmas and uncertainties, especially related with the capacity and protective intensity and his consequences in wide layers of the society. The article analyses the necessary elements to guarantee the sustainability of the public politics of welfare and suggests recommendations of adaptation to sociodemographic changes produced in the last stretch of the 20th century. 
Keywords: Social Intervention, Community Intervention, Mediation, Been ofWelfare, Systems of Social Protection, Social Services, Challenges, Sustainability.

\section{Introducción}

El trabajo que se presenta propone una reflexión acerca de la importancia de las prácticas de intervención comunitaria y de mediación en el conjunto de las políticas públicas ya que han supuesto un cambio considerable en la forma de llevar a cabo programas encaminados a mejorar la calidad de vida de las personas. Desde hace más de treinta años vienen formando parte del complejo entramado de prestaciones sociales que se dispensan en los distintos dispositivos de protección social. Durante este periodo se ha avanzado, no solamente en derechos con un importante entramado legislativo garantista, sino también en la calidad de la atención. Se han superado prácticas asistencialistas de carácter altruista, dando paso a respuestas profesionalizadas muy vinculadas a la arquitectura del nuevo mapa de prestaciones sociales que se ha generado con la modernización de las políticas públicas. El tránsito por los distintos sistemas de bienestar, de las prácticas de intervención comunitaria y de mediación aporta un balance positivo y se ha consolidado como uno de los elementos característicos de la atención social (Moreno, 2013).

Destaca especialmente la práctica comunitaria como una fórmula habitual dentro del Sistema Público de Servicios Sociales, participando en programas destinados a mejorar las condiciones de vida de familias en riesgo de exclusión. Se utiliza habitualmente en el trabajo con menores y forma parte de la lucha contra la pobreza y la precarización en distintos grupos poblacionales.

Pero las prácticas de intervención comunitaria no han sido exclusivas de los Servicios Sociales, también forman parte importante del Sistema Sanitario mediante el despliegue de actuaciones preventivas, especialmente en programas de salud mental, drogodependencias y otros trastornos aditivos, a través de prácticas de tratamiento y rehabilitación, fundamentadas en el reconocimiento de la existencia de determinantes sociales para la salud que hacen imprescindible la adopción de medidas compensatorias.

Por último cabe destacar la incidencia que han tenido numerosos programas y experiencias pertenecientes al Sistema Educativo en los que se han desple- 
gado programas de mediación con la finalidad de actuar en problemas prevalentes como el acoso escolar, mobbing, absentismo y otro tipo de conductas disruptivas dentro de la comunidad escolar (Fernández y Ponce de León, 2014: 229-231).

No obstante, la aportación más relevante de este tipo de prácticas no procede de actuaciones estanco, en cada uno de los sistemas de bienestar, sino que ha tenido que ver con aquella problemática social en la que interactúan distintos agentes y por tanto requiere de una mirada global, en estos casos, el sustrato comunitario de la intervención ha ofrecido avances importantes y logros destacables en la función paliativa, preventiva y también inclusiva.

A pesar de sus innegables aportaciones al conjunto de los sistemas de protección, el reto actual consiste en conocer el alcance de futuro de este tipo de prácticas, algo que va a estar muy ligado a la propia sostenibilidad del conocido como Estado de bienestar (EB). La capacidad de acoger prácticas que superen el mero asistencialismo forman parte de la esperanza respecto al futuro, no obstante, en el momento presente son tantos los dilemas a los que se enfrenta el futuro EB que nos ha parecido la mejor de las aportaciones, realizar un viaje hacia el pasado, conocer cuáles han sido las claves para modernizar la asistencia social, analizar los cambios sociodemográficos a los que se ha sometido la población española de finales del siglo XX y sobre todo, apuntar los principales retos e incertidumbres a los que se enfrenta la protección social en nuestro país, para terminar ofreciendo algunos elementos claves en la sostenibilidad y vigencia de futuro de los logros alcanzados.

\section{El Estado de bienestar como concepto, origen, fuentes y modelos}

El trayecto para la incorporación de España al moderno Estado del bienestar no ha sido fácil, ha estado salpicado de luces y de sombras, avances y retrocesos que han configurado un modelo de dispensación del bienestar en el que comparte una serie de elementos comunes con otros países meridionales.

Más allá de las dificultades por las que está atravesando en esos momentos, conviene resolver uno de los aspectos más relevantes que nos ayuda a comprender su impacto sobre la sociedad en su conjunto, el origen del EB con aquellos elementos que han coadyuvado a su aparición y su consolidación. 
Desde distintos posicionamientos se ha tratado de identificar, mediante una definición concreta aquello que se ha dado en llamar Estado del bienestar. Existe una cierta unanimidad para identificar la primera aparición de la expresión Estado de bienestar en 1942 en el conocido como Informe Beveridge. Dicho documento sirvió para que por primera vez se hablara de un Estado que adquiere compromisos respecto a las necesidades de los ciudadanos.

De cualquier forma delimitar el Estado de bienestar resulta complejo por diversos motivos, principalmente porque en cada país y en cada momento histórico la forma de proveer estos servicios propios del Estado han variado significativamente. Conviene hacer una salvedad, a pesar de que el término se acuñó en el año 1942, el EB es resultado de un proceso lento que se remonta a fínales del siglo XIX, y se encuentra íntimamente relacionado con la necesidad de dar respuesta a las carencias de una población y una sociedad en crecimiento y en continua evolución (Andrés, 2015).

Una primera aproximación sitúa como absolutamente indispensable la acción del sector público y la presencia constante de diversos instrumentos que permiten alcanzar un determinado nivel de vida garantizado al conjunto de los ciudadanos mediante la realización de distintas actuaciones, entre las que destacan las siguientes (Astelarra y Zaragoza, 2014):

Transferencias de renta para garantizar unos ingresos a los ciudadanos, ofertando servicios sociales bien directamente bien a través de organismos públicos o prestados por empresas privadas a cargo de los presupuestos del Estado, estableciendo subvenciones al consumo de determinados productos o servicios y promulgando leyes que regulen la calidad de los servicios públicos.

Además de los antecedentes enunciados, para obtener una definición concreta, resulta de enorme utilidad acudir a las señas de identidad y definir los elementos con los que se han ido configurando los distintos modelos y reconocer cuáles han sido sus consecuencias sobre los ciudadanos. De las distintas aproximaciones conceptuales, una de las que ha obtenido mayor consenso se corresponde con la efectuada por Ramesh Mishra en 1989 que define el EB como (Zaragoza, 2014b):

Sistema social desarrollado en las democracias capitalistas industrializadas, después de la Segunda guerra mundial y que tiene como finalidad favorecer el ejercicio de los 
derechos sociales de los ciudadanos e impulsar actuaciones públicas para reducir riesgos sociales.

La definición de Misrha recoge los elementos geográficos, históricos, políticos y económicos, que identifican una modalidad de dispensación de bienestar en la que interactúan tres agentes como son Estado, familia y mercado, adoptando cada uno de ellos una posición dominante en los distintos modelos que se han ido configurando a lo largo del siglo veinte.

Como indicábamos anteriormente no existe una modalidad exclusiva de EB, el sociólogo danés Gosta Esping-Andersen (2000) definió tres tipos: liberal, conservador y socialdemócrata, a los que atribuye una serie de características distintivas:

1. Modelo liberal anglosajón: en este caso el Estado decide cubrir únicamente los riesgos que no puede asumir una sociedad decente. Los riesgos se definen con carácter restrictivo y sólo se concede la protección social una vez comprobados los medios de vida e ingresos del beneficiario.

2. Modelo conservador-corporativista. Parte del principio de subsidiariedad. El Estado interviene siempre y cuando fallen las instituciones más próximas al individuo, como la familia.

3. Modelo socialdemócrata o nórdico, en el que el Estado cubre todos los riesgos partiendo del principio de universalidad de las prestaciones sociales. Supone la transformación de la asistencia social tradicional hacia un nuevo sistema con derechos universalizados.

El caso español no se corresponde con ninguna de las tipologías previas y encaja en un cuarto modelo que el mismo autor define como Mediterráneo, caracterizado por su menor intensidad, elevada carga familiarista, la existencia de sistema de dispensación mixta y por su aparición tardía en el ámbito europeo (Zaragoza, 2014a).

La evolución y expansión del EB español ha atravesado distintos momentos. El punto de arranque de las actuales políticas sociales hay que situarlo en la firma de los conocidos como Pactos de la Moncloa de 1977, suscritos por todos los partidos políticos con representación parlamentaria. En este primer periodo, vigente hasta 1981, se produce un importante avance en el reconocimiento de 
los derechos sociales en la Constitución de 1978, de forma que se origina un crecimiento considerable del gasto social, principalmente fruto de la extensión de las pensiones y de las prestaciones por desempleo.

La segunda etapa (1981-1989), se inicia en un momento de crisis y desempleo provocado por el tránsito de un modelo fordista hacia una sociedad postindustrial, esta segunda fase también produce importantes avances sociales como la completa extensión de la protección del desempleo mediante la puesta en marcha de las prestaciones de tipo asistencial.

En el periodo comprendido entre 1989 y 1995 se aumenta la cobertura del desempleo, surgen las Pensiones No Contributivas (PNC) y se universaliza la asistencia sanitaria. Todos estos avances suponen un elevado aumento del gasto social. En el año 1995 se lleva a cabo la firma del Pacto de Toledo que provoca un paréntesis en la situación de tensión social, consiguiendo un respaldo unánime de todos los partidos y del resto de agentes sociales al sistema público de pensiones. Unos años antes, gestionados por distintas Comunidades Autónomas se habían instaurado programas de renta mínima, siguiendo el modelo establecido en Francia de Revenu Minimum d'Insertiona (RMI).

La cuarta etapa recorre el periodo comprendido entre 1996 y 2001, se caracteriza por las negociaciones entre sindicatos y gobierno que favorecen la extensión del Estado del bienestar, aun a costa de reducir en parte su capacidad protectora (se contiene el gasto y se enduren algunas condiciones de cobro de pensiones) y se inicia un proceso de creciente privatización de servicios sanitarios educativos y sociales. En este periodo se racionaliza el sistema de la Seguridad Social (Ley 24/97) y se revalorizan las pensiones mínimas.

Por último conviene subrayar que el siglo XXI ha sido un periodo caracterizado más por recortes que por avances sociales. No obstante, hay que destacar el logro que supuso, la aprobación de la Ley 39/2006, de 14 de diciembre, de Promoción de la Autonomía Personal y Atención a las personas en situación de dependencia, más conocida como «ley de dependencia», mediante la cual se cierra el ciclo de la protección social con una Ley de vocación universalista que impone el derecho subjetivo como forma de acceso a las prestaciones para las personas dependientes y que opta por la profesionalización los cuidados, dos 
avances importantes que no han alcanzado los logros previstos, al menos con la intensidad esperada.

\section{Cambios sociodemográficos en la España del Siglo XXI}

El despliegue de las medidas protectoras se produce mientras que la sociedad española y la europea sufren una evolución importante en su composición y estructura sociodemográfica. Entre los fenómenos más relevantes se encuentran los siguientes:

- Cambios en el modelo de familia, el tránsito de la nuclear tradicional, extensa que había sido hegemónica hasta medias del siglo pasado se ha modificado sustancialmente, y se ha convertido en mayoritaria una fórmula que antes se consideraba atípica, caracterizadas por presentar menores tasas de fecundidad, constituida mediante emparejamientos menos estables y en las que ambos cónyuges forman parte del sustento familiar.

- Envejecimiento poblacional, algunos autores la han denominado como la mayor revolución del siglo XX, ya que ha aumentado cerca de diez años la perspectiva de vida y han aparecido nuevos tramos vitales, con importantes porcentajes de población afectada como la denominada cuarta edad.

- Crecimiento de familias en situación de exclusión y de pobreza con la aparición de desigualdades crecientes y el surgimiento de nuevos tipos de riesgo social intenso, como la pobreza energética, entre otros.

Las consecuencias de estos cambios afectan de manera considerable al desarrollo, implantación y niveles de éxito del denominado EB y son fundamentales a la hora de abordar el futuro y la sostenibilidad de los sistemas de protección social.

Pero estos cambios no han sido los únicos, también se han producido otros de tipo económico que modifican considerablemente las condiciones productivas de principios del siglo veinte, en las sociedades desarrolladas. Entre los más relevantes podemos destacar los siguientes: robotización, tecnologización, globalización de los mercados y terciarización del sistema productivo. 
La generalización de la nueva economía ha dificultado la expansión de los beneficios alcanzados, genera dificultades para el mantenimiento de los avances sociales y hace indispensable abrir un proceso de revisión de los actuales sistemas de bienestar.

\section{Algunos retos del sistema público de servicios sociales. Luces y sombras}

Conforme se apuntaba en el apartado anterior, el año 2006, concretamente en diciembre, se inicia una etapa nueva en la protección social en España con la promulgación de la que ha sido, al menos hasta el momento, la última aportación relevante en protección social para los ciudadanos, la conocida como Ley 39/2006, su despliegue, ha estado salpicado de diferentes dilemas y contradicciones. Posiblemente el mayor de ellos ha sido la coincidencia de su promulgación con el inicio de una de las etapas de crisis económica y social más intensa de la España moderna. Debido a esta circunstancia, el balance acerca de la utilidad de la Ley se tendrá que realizar desde la perspectiva de recuperación del pulso de normalidad de la economía española. Entre las luces aporta, como balance, un millón de personas atendidas, y entre las sombras hay que destacar los retrasos, las dificultades en la financiación y los trabas que han puesto algunas Comunidades para llevar a cabo el proceso de implantación.

Precisamente el carácter mixto de financiación y de gestión Autonómica ha supuesto una de las mayores dificultades, en algunos territorios ya que los resultados son enormemente desiguales. En diferentes Comunidades Autónomas, se puede considerar que ha quedado en entredicho el principio de derecho subjetivo que da sentido a la norma al estar condicionado por unos tiempos de tramitación que han puesto en cuestión su viabilidad como fórmula de acción social. También destaca, la ausencia de las prácticas preventivas previstas en el texto y sobre todo presenta un balance de enorme disparidad entre territorios.

Pero no es este el único problema al que se enfrenta la protección social en nuestro país, la situación de crisis económica ha puesto sobre la agenda política la necesidad de adoptar medidas más potentes en materia de lucha contra la exclusión social. Se ha planteado desde diferentes foros la necesidad de regular un sistema de Renta de Inserción de carácter estatal ya que hasta ahora se ha im- 
plantado bajo normativa autonómica que hace muy desigual el impacto y la calidad de la intervención. En el Parlamento español existen distintas iniciativas tendentes a regular esta prestación social e igualmente se ha abierto el debate acerca de la conveniencia de una Renta Básica como fórmula de garantía de ingresos. En esta misma línea de asignaturas pendientes se encuentra el crecimiento de la exclusión social y la pobreza, todos los informes recientes indican un aumento alarmante y la constatación de que las políticas públicas no han sido capaces de generar suficientes recursos de contención.

Para finalizar, este apartado de retos, cabría destacar la inexistencia de un plan integral o mapa de necesidades a nivel estatal, compatible con un Plan de Inclusión que garantice en el tiempo y de manera equitativa territorialmente la lucha contra la precarización social.

\section{Criterios de sostenibilidad}

Una vez analizado las dificultades conviene dirigir la mirada hacia el futuro e intentar atisbar cuál va a ser el panorama de la protección social especialmente confuso y controvertido, tratando de dilucidar acerca de la viabilidad de prácticas que han otorgado un papel relevante al Estado y han conseguido importantes niveles de calidad de vida a un número muy elevado de ciudadanos. La tarea no resulta sencilla debido a las dificultades que presentan los sistemas de bienestar y especialmente el de servicios sociales que se encuentra en un momento de construcción sometido a numerosos dilemas y debates, que dibujan un devenir plagado de incertidumbres.

Con la intención de realizar alguna aportación personal que pueda contribuir a despejar alguna de las dudas expuestas, planteamos un conjunto de criterios de sostenibilidad que consideramos indispensables para seguir avanzando en la durabilidad de los avances alcanzados. Este último apartado lo vamos a abordar desde una triple mirada, en función de las propuestas que se ofrecen, para ordenar las propuestas que se efectúan.

En primer lugar es indispensable una revisión de tipo económico, es un auténtico clamor la infrafinanciación de los distintos sistemas de protección social, en relación a los otros países de nuestro entorno. Todos los expertos destacan la 
insuficiente presencia del gasto social en el conjunto del gasto público, existe una distancia considerable a la media europea en este campo, al menos en comparación a países con un nivel de desarrollo equiparable al de España. Esta circunstancia viene agravada por la modalidad mixta de dispensación, la práctica totalidad del gasto social se gestiona desde las Comunidades Autónomas, solamente el sistema de pensiones dispone de una caja única en todo el Estado. Cada vez se hace más necesario revisar el modelo de financiación del gasto social y avanzar hacia lo que diferentes expertos plantean acerca de la conveniencia de utilizar cestas finalistas que blinden el destino final de cada partida presupuestaria, evitando que parte del gasto social acabe en otro tipo de gastos suntuarios que nada tienen que ver con el destino previsto. En este mismo apartado hay que evidenciar la importancia que tiene avanzar en la generación de una economía del bienestar que ofrezca sustento y seguridad mediante la creación de puestos de trabajo y fórmulas de colaboración con la Economía Social para hacer más eficiente la dispensación del bienestar.

En cuanto a los criterios sociales es una necesidad evidente profundizar en acuerdos y establecimiento de alianzas suficientes con agentes sociales, especialmente empleadores y los sindicatos, para favorecer la buena imagen de las políticas públicas de bienestar, incorporando a los nuevos movimientos sociales como elementos de respaldo de los avances sociales de finales del siglo pasado. Una estrategia para alcanzar este respaldo consiste, en llevar a cabo una política de servicios que sustituya paulatinamente la de prestaciones que puede resultar más sencilla de aplicar e incluso con mayor respaldo de los beneficiarios pero que no incorpora el valor añadido de los servicios mediante la creación de un importante número de puestos de trabajo que también otorgan credibilidad y respaldo social a los sistemas de bienestar.

Por último en cuanto a la sostenibilidad política ha llegado el momento de trasferir competencias fiscales a los territorios para que se corresponsabilicen realmente del resultado final del conjunto de las políticas públicas y especialmente de las políticas sociales. Además, hay que poner una mirada global a los principales retos y buscar garantías en todo el Estado en el cuarto de los Sistemas de bienestar, los servicios sociales ya que no se dispone de una Ley de ámbito estatal y se viene produciendo un incremento paulatino de síntomas de disparidad y de inequidad mediante un panorama cada vez más divergente. Para poner 
punto final a este apartado conviene destacar la necesidad de avanzar en la gobernanza europea y la relevancia de trasladar a todos los países de la Unión, elementos de compensación territorial, para establecer un sentimiento de pertenencia bajo un sistema de protección social con elementos equiparables.

\section{Conclusiones}

A la vista de la evolución alcanzada por el EB, agentes intervinientes y los cambios producidos, consideramos que las reformas que pueden dar más sostenibilidad a nuestro sistema de bienestar se han de mover en las siguientes claves:

- Fiscalidad suficiente, progresiva, transparente e inteligente. Debemos potenciar y prestigiar la fiscalidad como constructora de ciudadanía inclusiva y cohesión social.

- Austeridad. Hemos de reconocer que en el desarrollo de nuestro Estado de bienestar y en nuestras políticas públicas determinados responsables políticos han sido, en algunas ocasiones, frívolos, despilfarradores e irresponsables por clientelismo, imprevisión, ignorancia. No podemos volver a incurrir en dichos comportamientos.

- Activación. Toda medida de protección debe ir acompañada de las estrategias que potencien el protagonismo de la persona en la mejora de su situación y la corresponsabilidad. En el conjunto de la intervención social se debe potenciar la autoimplicación de la persona, en la medida de sus posibilidades.

- Flexibilidad. Los sistemas (sanidad, servicios sociales, garantía de ingresos...) han de articularse de forma flexible, de manera que sean capaces de adaptarse a diferentes coyunturas económicas, sociales... Para ello es fundamental trabajar en clave de innovación tecnológica, social y de introducción de fórmulas como la participación del tercer sector en la gestión de servicios, la creación de mercados sociales, nuevas fórmulas de mutualismo cívico y participación ciudadana, etc.

- Creación de empleo, riqueza y tejido social. Progresivamente hemos de ser capaces de ir sustituyendo los programas que se basan en la mera entre- 
ga de dinero en programas de prestación de servicios que son mucho más potentes para la creación de empleo, riqueza y tejido social.

- Buen gobierno, orden y rigor en la gestión y el control.

\section{Bibliografía}

ANDRÉS, S. (2015). Sociedad outlet-sociedad low cost. La clase media vuelve a casa. Ehquidad. International Welfare Policies and Social Work Journal, 4, 11 - 30. Doi:10.15257/ ehquidad.2015.0007

ASTELARRA, J., y ZARAGOZA, G. (2014). El Estado de Bienestar en España. Criterios de sostenibilidad. Ehquidad. InternationalWelfare Policies and SocialWork Journal, 1, 61-90. Doi: 10.15257/ehquidad.2014.0003

ESPING-ANDERSEN, G. (2000). Fundamentos Sociales de las Economías Postindustriales. Madrid: Ariel.

FERNÁNDEZ GARCÍA, T., y PONCE DE LEÓN, L. (2014). Nociones básicas de Trabajo Social. Madrid: Ediciones Académicas.

MORENO, L. (2013). La Europa Asocial. Madrid: Península.

ZARAGOZA PASCUAL, G. (2014a). De la protección Social al Estado de Bienestar. Revista de Treball, Economia i Societat. (CES), 72, 21.

ZARAGOZA PASCUAL, G. (2014b). Crónica de Bienestar en tiempos de malestar. Valencia: PUV Universidad de Valencia. 